\title{
Sigmoid colon cancer arising in a diverticulum of the colon with involvement of the urinary bladder: a case report and review of the literature
}

\author{
Yasumichi Yagi ${ }^{1 *}$, Yasuhiro Shoji ${ }^{1}$, Shozo Sasaki ${ }^{1}$, Akemi Yoshikawa', Yuji Tsukioka', Wataru Fukushima', \\ Hisashi Hirosawa ${ }^{1}$, Ryohei Izumi ${ }^{1}$ and Katsuhiko Saito ${ }^{2}$
}

\begin{abstract}
Background: Colon cancer can arise from the mucosa in a colonic diverticulum. Although colon diverticulum is a common disease, few cases have been previously reported on colon cancer associated with a diverticulum. We report a rare case of sigmoid colon cancer arising in a diverticulum with involvement of the urinary bladder, which presented characteristic radiographic images.

Case presentation: A 73-year-old man was admitted to our hospital for macroscopic hematuria. Computed tomography and magnetic resonance imaging revealed a sigmoid colon tumor that protruded into the urinary bladder lumen. The radiographs showed a tumor with a characteristic dumbbell-shaped appearance. Colonoscopy showed a type 1 cancer and multiple diverticula in the sigmoid colon. A diagnosis of sigmoid colon cancer with involvement of the urinary bladder was made based on the pathological findings of the biopsied specimens. We performed sigmoidectomy and total resection of the urinary bladder with colostomy and urinary tract diversion. Histopathological findings showed the presence of a colovesical fistula due to extramurally growing colon cancer. Around the colon cancer, the normal colon mucosa was depressed sharply with lack of the muscular layer, suggesting that the colon cancer was arising from a colon diverticulum.

Conclusion: The present case is the first report of sigmoid colon cancer arising in a diverticulum with involvement of the urinary bladder. Due to an accurate preoperative radiological diagnosis, we were able to successfully perform a curative resection for sigmoid colon cancer arising in a diverticulum with involvement of the urinary bladder.
\end{abstract}

Keywords: Colon cancer, Diverticulum, Urinary bladder invasion

\section{Background}

Colon cancer can arise from the mucosa in a colonic diverticulum. Although colon diverticulum is a common disease, few cases have been previously reported on colon cancer associated with a diverticulum [1-8]. Because a diverticulum lacks the muscular layer, cancerous tissue arising within a diverticulum can easily penetrate the serosa as it grows and may not be detected until an advanced stage [4-6]. Moreover, the specific progression with intramural growth may make it difficult to exactly diagnose. Herein, we report a rare case of

\footnotetext{
* Correspondence: y-yagi@live.jp

'Department of Surgery, Toyama City Hospital, 2-1 Imaizumi Hokubu-machi, Toyama 939-8511, Japan

Full list of author information is available at the end of the article
}

sigmoid colon cancer arising in a diverticulum with involvement of the urinary bladder, which was accurately diagnosed by radiological images.

\section{Case presentation}

A 73-year-old man was admitted to our hospital for macroscopic hematuria. Enhanced abdominal computed tomography $(\mathrm{CT})$ revealed a sigmoid colon tumor which protruded into the urinary bladder lumen. Neither nodal metastasis nor distant metastasis was detected. Enhanced abdominal magnetic resonance imaging (MRI) also revealed a solid tumor in the sigmoid colon and the urinary bladder, both of which were connected to each other by a fistula. Both the CT and the MRI presented a characteristic dumbbell-shaped appearance (Figure 1).

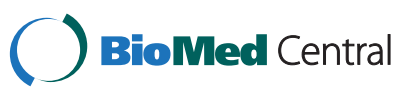




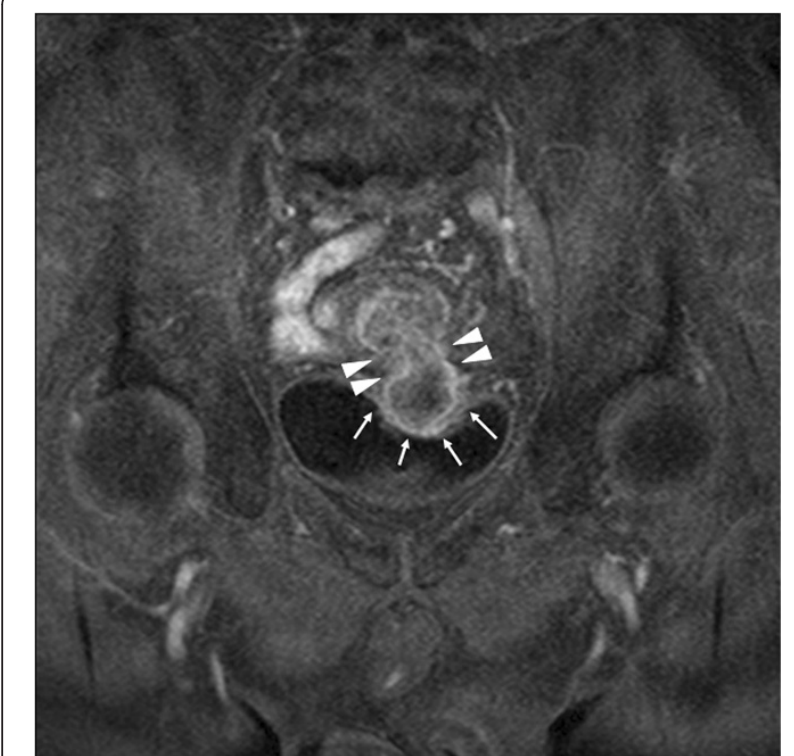

Figure 1 Enhanced MRI T1-weighted image. Between the sigmoid colon and the urinary bladder, the solid tumor was connected via the fistula, showing a dumbbell-shaped appearance. The sigmoid colon tumor protruded into the urinary bladder lumen (arrows) via the colovescal fistula (arrowheads).

Colonoscopy showed a type 1 tumor and multiple diverticula in the sigmoid colon. The pathological diagnosis of the biopsied sigmoid colon tumor was well-differentiated tubular adenocarcinoma. Barium enema examination showed a tumor and multiple diverticula in the sigmoid colon, but could not clarify a communication with the urinary bladder lumen (Figure 2). Cystoscopic examination showed a tumor in the urinary bladder lumen. The pathological diagnosis of the biopsied bladder tumor was well-differentiated tubular adenocarcinoma, not transitional cell cancer, deriving from the sigmoid colon cancer. The laboratory data, including inflammatory reaction and tumor markers, were within normal limits. On the other hand, occult hematuria was detected due to the urinary bladder tumor. Thereafter, the diagnosis was established as sigmoid colon cancer with involvement of the urinary bladder (cT4N0M0, cStageIIB according to the UICC TNM classification). The therapeutic strategy was explained to the patient, who decided to undergo a surgical resection. We performed sigmoidectomy and total resection of the urinary bladder with colostomy and urinary tract diversion. The postoperative period was uneventful. Pathological examination of the resected specimen revealed well-differentiated tubular adenocarcinoma without nodal metastasis of the regional lymph node (pT4N0M0, pStageIIB), confirming the preoperative diagnosis. Macroscopic cross section of the resected specimen exhibited a dumbbell-shaped appearance, just as was shown in the MRI findings (Figure 3). Histopathological findings showed

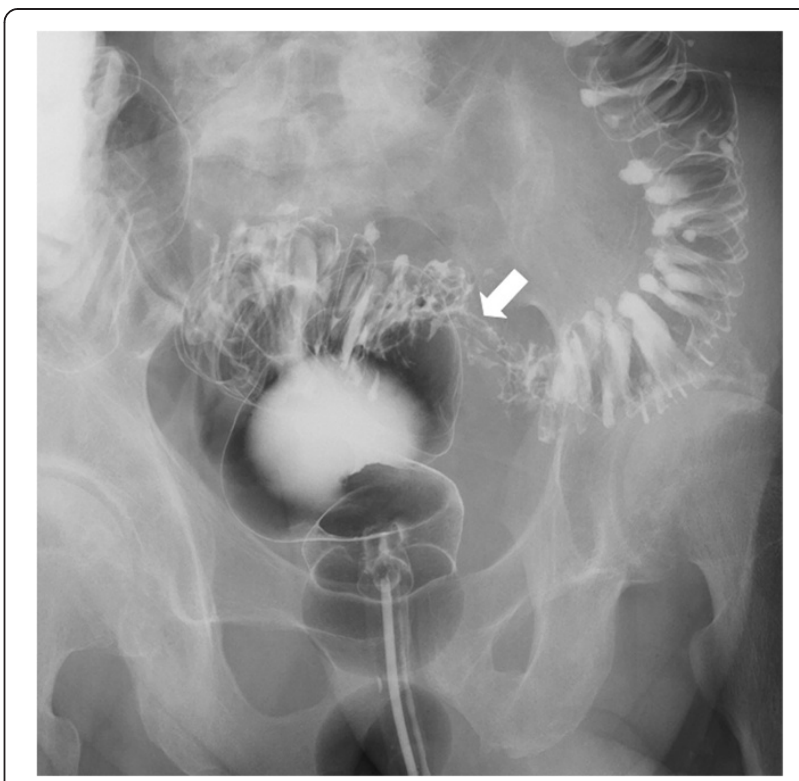

Figure 2 Barium enema findings. The colon tumor was detected as a defect in the sigmoid colon (arrow). Around the sigmoid colon cancer, there were multiple colon diverticula in the sigmoid colon.

the presence of a colovesical fistula due to extramurally growing colon cancer. However, the intraperitoneal space was not exposed to cancerous tissue because the colovesical fistula was completely covered with a fibrous tissue layer (Figure 4A). In addition, around the colon cancer, the normal colon mucosa was depressed sharply with a thinwalled muscular layer, suggesting the presence of a colon diverticulum. The cancerous tissue protruded from the base of the inverted mucosa of the diverticulum to the colovesical fistula. The surrounding muscular layer was not involved in the process of the tumor progression. Thereafter, it was demonstrated that the colon cancer had arisen from the mucosa of the colon diverticulum (Figure 4B). The unique radiological findings of the present case were based on the histological findings of the progression via the colovesical fistula derived from a colon diverticulum. No evidence of recurrence was found at 12 months follow-up.

\section{Discussion}

Cancer may incidentally arise in a colonic diverticulum. Based on an endoscopic finding of a tumor within a diverticulum, a diagnosis of early colon cancer arising in a diverticulum can be made $[3,5,8]$. In regard to cases of advanced colon cancer, the diagnosis mostly depends on the histological findings of the resected specimen, except for a tumor arising in a large diverticulum. However, diagnosis may be complicated by abnormal findings, such as abscess formation, submucosal progression, and diverticulitis [2-4]. In the present case, the pathological examination exhibited characteristic findings that the 


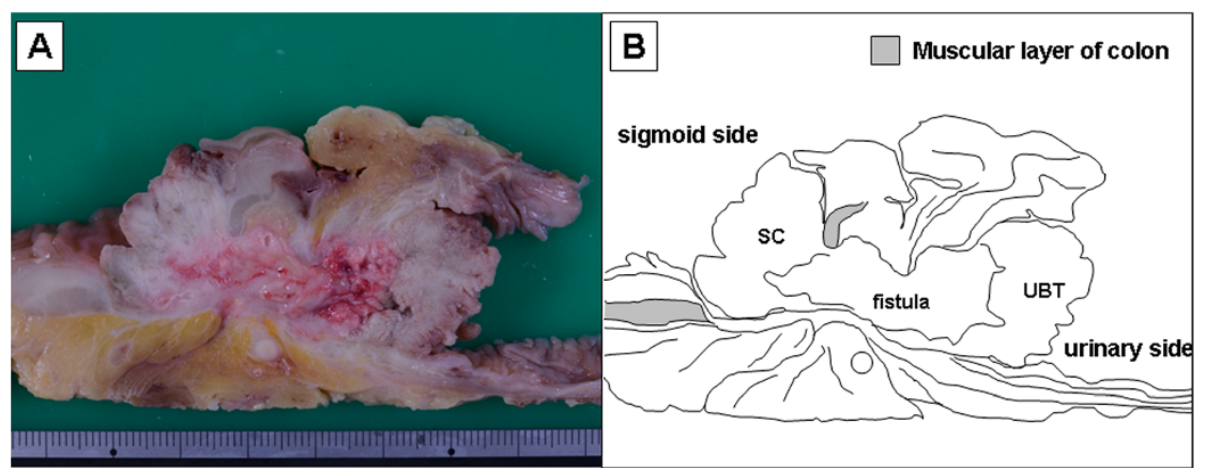

Figure 3 Cross section of the resected specimen. A: Macroscopic picture of a cross section. B: Diagram of a cross section. The progression of the sigmoid colon cancer was reflected in the MRI findings. The macroscopic findings revealed the progression via the colovesical fistula. SC: sigmoid colon cancer. UBT: urinary bladder tumor.

primary tumor of the sigmoid colon progressed via the colovesical fistula without peritoneal exposure. Moreover, the fistula was continuous with the inverted colon mucosa of the diverticulum. On the basis of these microscopic findings, the sigmoid colon cancer progressed along the structure of the fistula which was supposed to arise from the diverticulitis of the sigmoid colon since before the genesis of the sigmoid colon cancer. The irregular progression was plainly reflected in the radiological finding of a dumbbell-shaped tumor.

The sigmoid colon and rectum are common sites of a primary tumor invading the urinary bladder compared with other colon segments $[9,10]$. Previous reports have demonstrated this; even in cases of local advanced colorectal cancer with a colovesical fistula, extended surgery with en-bloc bladder resection contributes to local control and improvement of survival $[11,12]$. In short, the prognosis depends on the negative surgical margin and the status of the nodal metastasis with or without a colovesical fistula $[13,14]$. However, the extended resection requires partial or total resection of the bladder with urinary tract diversion. In the present case, it was inadequate to preserve the bladder, because the tumor invaded the triangle of the bladder. If the tumor was located apart from the triangle of the bladder, it should be considered to preserve the bladder with the partial resection of the bladder. Thereafter, precise radiological diagnosis preoperatively is recommended in order to properly determine the extent of the surgical resection.

According to previous reports, there have been ten cases of colon cancer arising in a diverticulum [1-8]. Of these ten cases, two were early cancer, and the other eight were advanced stage. As for the location of the lesion, in three cases it was the right side of the colon, and in the other seven it was the left side of the colon. In two cases, the cancer was associated with diverticulitis. In the present case, it was not clear whether the cancer was associated with diverticulitis or fistula due to diverticulitis. From the

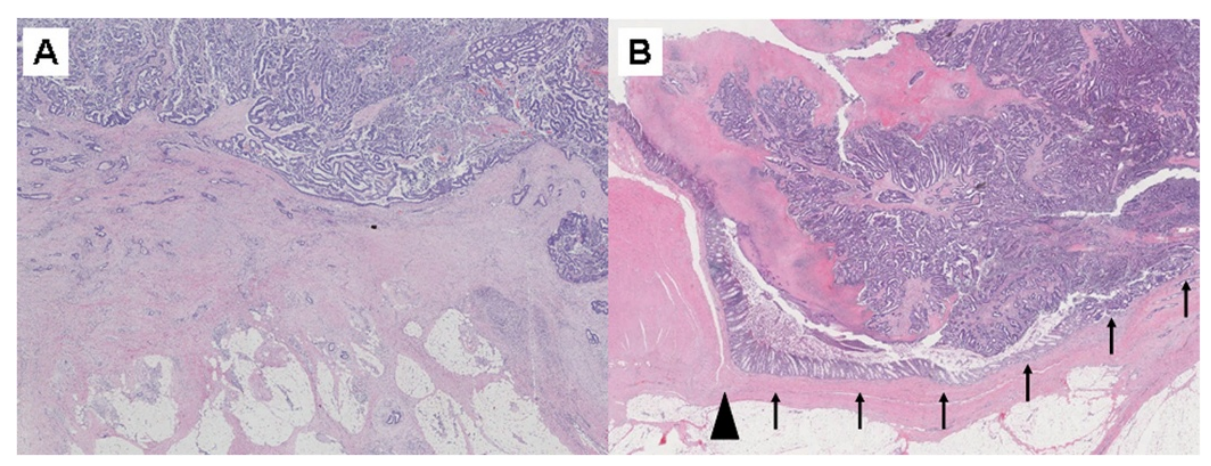

Figure 4 Microscopic findings of the resected specimen (H-E staining, $\times \mathbf{2 0 0})$. A: The sigmoid colon cancer, penetrating to the urinary bladder, did not invade the surrounding muscular layer as the tumor progressed. Adjacent to the sigmoid tumor, the normal colon mucosa was depressed sharply (arrows) with a thin-walled muscular layer (arrowhead), indicating that the colon cancer had arisen from a colon diverticulum. B: The sigmoid and urinary sides of the tumor were connected via the colovesical fistula. In the fistula, the cancerous tissue was completely covered with fibrous tissue, indicative of no exposure to the intraperitoneal space to cancerous tissue. 
patient's history, the communication between the colon and the bladder lumen might be temporally formed with inflammation of the bladder. However, the fistula might not be necessarily certified at this advanced stage after the tumor occupied the lumen of both the colon and the bladder. To the best of our knowledge, the present case is the first report of sigmoid colon cancer arising from a diverticulum with involvement of the urinary bladder.

\section{Conclusion}

In conclusion, due to an accurate preoperative radiological diagnosis, we were able to successfully perform a curative resection for sigmoid colon cancer arising in a diverticulum with involvement of the urinary bladder.

\section{Consent}

Written informed consent was obtained from the patient for publication of this case report and any accompanying images. A copy of the written consent is available for review by the Editor-in-Chief of this journal.

\section{Competing interests}

The authors declare that they have no competing interests in this paper.

\section{Authors' contributions}

YY edited the manuscript. YS, SS, AY, YT, WF, HH, and RI supervised the whole study. KS provided advice on the pathological findings. All authors read and approved the final manuscript.

\section{Acknowledgement}

We thank Koichi Kodama and yasukazu Takase (Department of Urology, Toyama City Hospital) for discussion and suggestions about the diagnosis of this case.

\section{Author details}

'Department of Surgery, Toyama City Hospital, 2-1 Imaizumi Hokubu-machi, Toyama 939-8511, Japan. Department of Pathology, Toyama City Hospital,

2-1 Imaizumi Hokubu-machi, Toyama 939-8511, Japan.

Received: 18 January 2014 Accepted: 7 May 2014

Published: 13 May 2014

\section{References}

1. Drut R: Adenoacanthoma arising in a diverticulum of the colon: report of a case. Dis Colon Rectum 1974, 17(2):258-261.

2. Hines JR, Gordon RT: Adenocarcinoma arising in a diverticular abscess of the colon: report of a case. Dis Colon Rectum 1975, 18(1):49-51.

3. McCraw RC, Wilson SM, Brown FM, Gardner WA: Adenocarcinoma arising in a sigmoid diverticulum: report of a case. Dis Colon Rectum 1976, 19(6):553-556

4. Cohn KH, Weimar JA, Fani K, DeSoto-LaPaix F: Adenocarcinoma arising within a colonic diverticulum: report of two cases and review of the literature. Surgery 1993, 113(2):223-226.

5. Kajiwara H, Umemura S, Mukai M, Sadahiro S, Tsutsumi Y: Adenocarcinoma arising within a colonic diverticulum. Pathol Int 1996, 46(7):538-539.

6. Kikuchi T, Kotanagi H, Kon H, Koyama K, Ito S, Otaka M: Mucosal carcinoma within a colonic diverticulum. J Gastroenterol 1999, 34(5):622-625.

7. Bellows CF, Haque S: Adenocarcinoma within a diverticulum: a common tumor arising in an uncommon location. Dig Dis Sci 2002, 47(12):2758-2759.

8. Fu Kl, Hamahata Y, Tsujinaka Y: Early colon cancer within a diverticulum treated by magnifying chromoendoscopy and laparoscopy. World J Gastroenterol 2010, 16(12):1545-1547.

9. Kobayashi T, Kamoto T, Sugino $Y$, Takeuchi H, Habuchi T, Ogawa O: High incidence of urinary bladder involvement in carcinomas of the sigmoid and rectum: a retrospective review of 580 patients with colorectal carcinoma. J Surg Oncol 2003, 84(4):209-214.

10. Talamonti MS, Shumate CR, Carlson GW, Curley SA: Locally advanced carcinoma of the colon and rectum involving the urinary bladder. Surg Gynecol Obstet 1993, 177(5):481-487.

11. Hotta T, Takifuji K, Yokoyama S, Matsuda K, Higashiguchi T, Tominaga T, Aoki Y, Taniguchi K, Yamaue H: Survival in colorectal cancer patients with urinary tract invasion. Dis Colon Rectum 2006, 49(9):1399-1409.

12. Gao F, Cao YF, Chen LS, Zhang S, Tang ZJ, Liang JL: Outcome of surgical management of the bladder in advanced colorectal cancer. Int J Colorectal Dis 2007, 22(1):21-24.

13. Winter DC, Walsh R, Lee G, Kiely D, O'Riordain MG, O'Sullivan GC: Local involvement of the urinary bladder in primary colorectal cancer: outcome with en-bloc resection. Ann Surg Oncol 2007, 14(1):69-73.

14. Curley SA, Carlson GW, Shumate CR, Wishnow Kl, Ames FC: Extended resection for locally advanced colorectal carcinoma. Am J Surg 1992 163(6):553-559.

doi:10.1186/1471-230X-14-90

Cite this article as: Yagi et al:: Sigmoid colon cancer arising in a

diverticulum of the colon with involvement of the urinary bladder: a case report and review of the literature. BMC Gastroenterology 2014 14:90.

\section{Submit your next manuscript to BioMed Central and take full advantage of:}

- Convenient online submission

- Thorough peer review

- No space constraints or color figure charges

- Immediate publication on acceptance

- Inclusion in PubMed, CAS, Scopus and Google Scholar

- Research which is freely available for redistribution 